\title{
Postoperative Lead Movement after Deep Brain Stimulation Surgery and the Change of Stimulation Volume
}

\author{
Nathanael Göransson ${ }^{a, b}$ Johannes D. Johansson ${ }^{a, c}$ Karin Wårdell ${ }^{a, c}$ \\ Peter Zsigmond ${ }^{\mathrm{b}}$

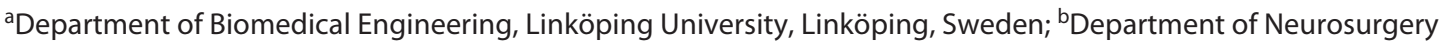 \\ and Department of Biomedical and Clinical Sciences, Linköping University, Linköping, Sweden; ' Center for Medical \\ Image Science and Visualization, Linköping University, Linköping, Sweden
}

\section{Keywords}

Deep brain stimulation - Displacement - Electric field ·

Functional neurosurgery · Movement disorder surgery

\begin{abstract}
Introduction: Lead movement after deep brain stimulation may occur and influence the affected volume of stimulation. The aim of the study was to investigate differences in lead position between the day after surgery and approximately 1 month postoperatively and also simulate the electric field (EF) around the active contacts in order to investigate the impact of displacement on affected volume. Methods: Twenty-three patients with movement disorders underwent deep brain stimulation surgery (37 leads). Computed tomography at the 2 time points were co-fused respectively with the stereotactic images in Surgiplan. The coordinates $(x$, $y$, and $z$ ) of the lead tips were compared between the 2 dates. Eleven of these patients were selected for the EF simulation in Comsol Multiphysics. Postoperative changes of EF spread in the tissue due to conductivity changes in perielectrode space and due to displacement were evaluated by calculating the coverage coefficient and the Sørensen-Dice coeffi-
\end{abstract}

cient. Results: There was a significant displacement (mean \pm SD) on the left lead: $x(0.44 \pm 0.72, p<0.01), y(0.64 \pm 0.54$, $p<0.001)$, and $z(0.62 \pm 0.71, p<0.001)$. On the right lead, corresponding values were: $x(-0.11 \pm 0.61, \mathrm{~ns}), y(0.71 \pm 0.54$, $p<0.001)$, and $z(0.49 \pm 0.81, p<0.05)$. The anchoring technique was a statistically significant variable associated with displacement. No correlation was found between bilateral $(n=14)$ versus unilateral deep brain stimulation, gender $(n=$ 17 male), age $<60$ years $(n=8)$, and calculated air volume. The simulated stimulation volume was reduced after 1 month because of the perielectrode space. When considering perielectrode space and displacement, the volumes calculated the day after surgery and approximately 1 month later were partly overlapped. Conclusion: The left lead tip displayed a tendency to move lateral, anterior, and inferior and the right a tendency to move anterior and inferior. The anchoring technique was associated to displacement. New brain territory was affected due to the displacement despite considering the reduced stimulated volume after 1 month. Postoperative changes in perielectrode space and small lead movements are reasons for delaying programming to 4 weeks following surgery.

(c) 2020 The Author(s)

Published by S. Karger AG, Basel karger@karger.com www.karger.com/sfn

Karger $\stackrel{\text { ' }}{5}$

BOPEN ACCESS
(C) 2020 The Author(s)

Published by S. Karger AG, Basel

This is an Open Access article licensed under the Creative Commons Attribution-NonCommercial-4.0 International License (CC BY-NC) (http://www.karger.com/Services/OpenAccessLicense), applicable to the online version of the article only. Usage and distribution for commercial purposes requires written permission.
Nathanael Göransson

Department of Neurosurgery and Department of Biomedical and Clinical Sciences Linköping University Hospital

SE-581 85 Linköping (Sweden)

nathanael.goransson@ regionostergotland.se 


\section{Introduction}

Deep brain stimulation (DBS) surgery has been proven to be an effective method in the symptomatic treatment of movement disorders such as Parkinson's disease (PD), essential tremor (ET), and dystonia [1-3]. It is also used to explore the therapeutic effect in different neuropsychiatric disorders $[3,4]$. The treatment efficacy and absence of adverse effect is highly dependent on the electrode position [2, 5-7]. Previous studies have reported lead displacement that occurs postoperatively [8-11]. The postoperative displacement have been attributed to technical errors, failure of lead fixation, cerebrospinal fluid loss, pneumocephalus, twiddler syndrome, and dystonia $[8,9,11]$. A marginal postoperative movement may affect the clinical outcome after surgery since the target area is small, that is, in the range of millimeters $[5,12]$. Therefore, the maintenance of electrode position in the target area is essential for good outcome [13].

Patient-specific simulation of the electric field (EF) surrounding the DBS lead is a method to investigate the affected tissue $[14,15]$. The EF can be superimposed on the patient's MR images, thus visualizing the influenced anatomy of the individual patient. Examples of studies where patient-specific simulations have been used are investigation of speech intelligibility in $\mathrm{PD}$ [16], delineation of thalamic structures for ET stimulation [17], targeting of the limbic globus pallidus internus (GPi) in Tourette syndrome [18], and variations in lead design [15]. Thus, the impact of a lead displacement can be evaluated using patient-specific simulation.

A notable early clinical symptom relief in some patients is attributed to the microlesion effect from the trauma after lead insertion. This clinical effect is present regardless of stimulation and recedes with time $[19,20]$. The trauma of the DBS electrode implantation in the brain will also trigger a small inflammatory response, forming a perielectrode edema. Higher water content in the edematic tissue increases the electric conductivity. With time, the edema recedes and gliosis occurs in the perielectrode space (PES), reducing the electric conductivity. The higher electrical conductivity in the early postoperative stage therefore increases the extension of the EF spread around the electrode compared to the chronic state, that is, a month after implantation [21-23]. Previous simulation studies have also shown that the EF spreads differently depending on the tissue type due to different electric conductivity properties [15]. This is particularly notable in the presence of CSF-filled cysts [24].
The aim of the present study was to investigate postoperative lead displacement after approximately 1 month and to study possible contributing factors such as age, diagnosis, bilateral/unilateral implantation, postoperative air volume, and lead anchoring. Postoperative changes of the EF spread in the tissue due to displacement and changes in PES conductivity were evaluated at the 2 time points, directly after (acute) and 1 month post-implantation (chronic) in order to investigate their impact on stimulated surrounding volume.

\section{Material and Methods}

\section{Patients}

Twenty-three patients (17 male and 6 female, mean \pm SD age 61 \pm 10 years) were operated with DBS at the Department of Neurosurgery, Linköping University Hospital, between January 2014 and February 2016. The population included 11 ET, 10 PD, 1 Parkinson tremor, and 1 patient with Dystonia. A total of 37 leads were implanted, 14 bilateral, and 9 unilateral. All unilateral leads were placed on the left side. Targets for DBS treatment were zona incerta $(n=$ $11)$, ventral intermediate nucleus of the thalamus $(n=8)$, subthalamic nucleus $(n=16)$, and GPi $(n=2)$. Bilateral implantations were counted as 2 leads for target numbers. The study was approved by the local Ethics Committee (2012/434-31) and all patients gave their informed written consent to participate in the study.

\section{Surgery}

Prior to surgery, the stereotactic frame (Leksell Stereotactic System, Elekta Instruments AB, Stockholm, Sweden [LSS]) was mounted on the head. Surgery was performed during general anesthesia using direct visualization of targets (subthalamic nucleus, zona incerta, and GPi) with SurgiPlan (Elekta Instruments $\mathrm{AB}$, Stockholm, Sweden). Coordinates from the SchaltenbrandWahren human brain atlas was utilized for indirect targeting of ventral intermediate nucleus of the thalamus. The DBS surgery was performed for all patients in 1 stage. The head elevation was approximately $20-30^{\circ}$ in a semirecumbent position. Burr holes were made at the level of the coronal suture $2-3 \mathrm{~cm}$ lateral from the midline. Tisseel glue (Baxter Medical AB, Sweden) was placed in the burr hole after opening the dura to minimize the CSF loss and air entry. Under fluoroscopic visualization (Philips BV Pulsera, Philips Medical Systems, Best, The Netherlands) the quadripolar DBS electrode lead 3389 (Medtronic, Minneapolis, MN, USA) was implanted. The left lead was always implanted first. The leads were secured in the burr hole by using either the anchoring cover that is boxed with the lead, standard anchoring $(n=24)$ (Medtronic, Minneapolis, MN, USA), Stimloc ${ }^{\mathrm{TM}}$ Burr Hole Cover $(n=9)$ (Medtronic, Minneapolis, MN, USA) or with the anchoring device from Guardian ${ }^{\mathrm{TM}}$ Burr Hole Cover $(n=4)$ (St. Jude Medical Inc., Little Canada, MN, USA). The extension kit 37086 (Medtronic, Minneapolis, MN, USA) and the implantable pulse generator were subcutaneously placed in the same procedure. Activa PC 37601 (Medtronic, Minneapolis, MN, USA) was used for bilateral stimulation and Activa SC 37603 (Medtronic, Minneapolis, MN, USA) for unilateral stimulation. The surgical procedure is described in previous studies $[25,26]$ and usually took $4-5 \mathrm{~h}$. 


\section{Imaging}

Preoperative MRI examination using axial slices, $0.49 \times 0.49 \times$ $2 \mathrm{~mm}$, on either a $3 \mathrm{~T}$ (Ingenia, Philips Healthcare, Best, The Netherlands) or a $1.5 \mathrm{~T}$ (Achieva, Philips Healthcare, Best, The Netherlands) system was performed. MRI were either with LSS $(n=16$ [3 $\mathrm{T}: n=13,1.5 \mathrm{~T}: n=3])$ or frameless $(n=7)$. In patients examined with frameless preoperative MR a 3D CT imaging with voxel size $0.55 \times 0.55 \times 1 \mathrm{~mm}$, with the use of a 128-slice CT scanner (Somatom Definition AS, Siemens, Forchheim, Germany) was done with LSS. The stereotactic CT with frame was co-registered to the frameless MRI before planning of targets and trajectories in SurgiPlan. The day after surgery and after $33 \pm 6$ days (mean \pm SD), a postoperative high definition CT was taken using the same CT scan protocol as previously described.

\section{Definition of Final Lead Position}

In order to define the final position of the leads, the 2 postoperative CT images were co-registered in SurgiPlan respectively with the MR or CT images preoperatively taken with the stereotactic frame and aligned to the AC-PC-line. The lead tip, center of the bottom edge of contact 0, was used as the reference point. Each electrode was visualized with appropriate windowing by adjusting the level in SurgiPlan. The Leksell coordinates $(x, y, z)$ of the lead tip on the 2 postoperative images were defined. Lead movement was measured by comparing the coordinates on the distal contact between the CT images taken at the 2 postoperative time points, acute and chronic. The displacement in millimeter was defined as the coordinate at the chronic time point minus the coordinate at the acute time point giving a displacement in $x, y$, and $z$ directions. Eleven of these patients were selected for the EF simulation part. These patients fulfilled technical requirement of a high quality preoperative $3 \mathrm{~T}$ MRI with frame and active electrode settings after at least 1 month. There were 2 additional patients with 3 T MRI and frame. One had suboptimal image quality for simulation and 1 patient suffered from an early infection and the DBS system was removed before starting. These 2 patients were therefore excluded for the EF simulation part. In the simulation group the coordinates of the lead $10 \mathrm{~mm}$ above AC-PC was also noted for the 2 postoperative time points. This gave 2 positions per time point, the tip and $10 \mathrm{~mm}$ above AC-PC along the lead. These coordinates were used to place the DBS lead in a patient-specific brain model $[15,27]$.

\section{Patient-Specific Simulation of Electric Field}

Models of the Medtronic 3389 lead were made in Comsol Multiphysics 5.2 (Comsol AB, Sweden). The electric field magnitude EF was calculated from the equation for steady currents with the electric conductivity taken from tissue classification with in-house software ELMA $[15,27,28]$ of the T2-weighted preoperative $3 \mathrm{~T}$ MRI. The tissue was classified into gray matter, white matter, blood, or CSF, and their conductivities were assigned from tabulated values $[29,30]$. The negative contacts were assigned the electric potential used for the individual patients while the surrounding surfaces were set to ground for monopolar stimulation. For bipolar stimulation, the positive contacts were assigned to ground instead.

Brain models were made for the acute and chronic stages with and without inclusion of the PES. For the latter case a PES of 250 $\mu \mathrm{m}[22]$ was assumed to have an electric conductivity of $0.14 \mathrm{~S} / \mathrm{m}$ in the acute phase and $0.05 \mathrm{~S} / \mathrm{m}$ in the chronic phase based on model adaptions [23] to impedance measurements by Codrin
Table 1. Stimulation parameters of the patients with EF simulations

\begin{tabular}{lcc}
\hline Parameter & Mean \pm SD & Range \\
\hline Voltage & $2.2 \pm 1 \mathrm{~V}$ & $0.2-3.8 \mathrm{~V}$ \\
Pulse frequency & $133 \pm 8 \mathrm{~Hz}$ & $130-160 \mathrm{~Hz}$ \\
Pulse width & $66 \pm 14 \mu \mathrm{s}$ & $60-100 \mu \mathrm{s}$ \\
Contacts & $1.7 \pm 0.8$ & $1-3$ \\
Monopolar/bipolar & $15 / 3$ & \\
Bilateral/unilateral & $7 / 4$ & \\
\hline
\end{tabular}

EF, electric field.

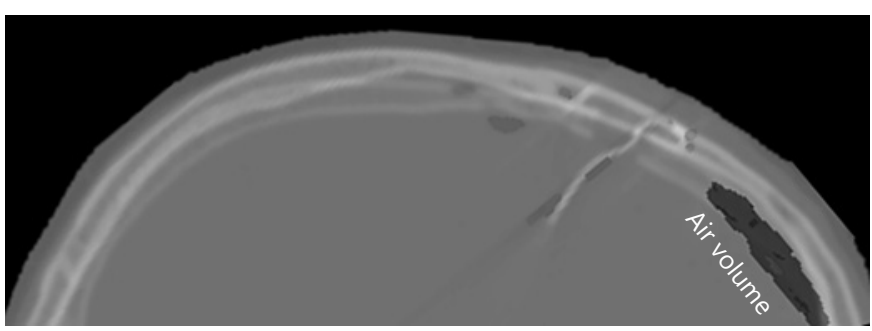

Fig. 1. Sagittal plane of a skull. Demonstrating the postoperative air located in the frontal subdural space anterior to the electrode.

Lungu et al. [31]. Within the PES, conductivity values of the PES replaced the tissue classification conductivity from the tabulated values. The electrodes were aligned by the 2 coordinates along the lead, the tip and $10 \mathrm{~mm}$ above AC-PC, at the 2 time points respectively. Simulations were done using the latest clinical settings, all in the chronic stage. For detailed description of modeling and simulation see Åström et al. [14], Alonso et al. [15], and Johansson et al. [23].

The threshold electric field magnitude $\mathrm{EF}_{\mathrm{t}}$ required for stimulation of the axons in the surrounding tissue of the lead was assumed to be $0.2 \mathrm{~V} / \mathrm{mm}$ for the 9 patients with the pulse width of $60 \mu$ s. This threshold has been used in several previous studies [15-17]. Increasing the pulse width lowers the $\mathrm{EF}_{\mathrm{t}}$ and consequently also the voltage required for clinical effect. For the 2 patients with larger than $60 \mu$ s pulse width, we used $0.17 \mathrm{~V} / \mathrm{mm}$ for the pulse width of $90 \mu$ s and $0.16 \mathrm{~V} / \mathrm{mm}$ for the pulse width of 100 $\mu$ s respectively according to strength-duration measurements by Mario Rizzone et al. [32]. These values have been found to be consistent with experimental estimation of the stimulation spatial extent by Alexis Kuncel et al. [33]. The stimulation parameters for the simulated patients are presented in Table 1. For details, see reference $[23,34]$.

\section{Air Volume Calculation}

All the patients were considered for volume analysis. As the volume always was zero in the nonoperated side, the air was only calculated in the hemisphere where the lead was implanted. The air volumes on the left side and right side were categorized separately and in total. The air locations were first identified in the Sante DICOM Viewer program (Santesoft Ltd., Nicosia, Cyprus) 


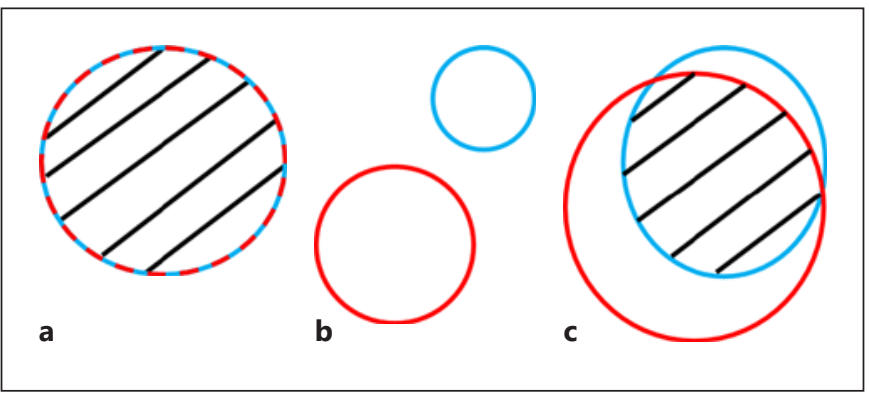

Fig. 2. The common volume of 2 volumes used for $D_{\mathrm{C}}$ and $C_{\mathrm{C}}$. Red is the acute volume, blue is the chronic volume. Example is given in the axial plane, showing anterior and lateral movement. a The 2 volumes show a complete overlap, $D_{C}$ and $C_{C}=1$. b The 2 volumes are completely separated, $D_{\mathrm{C}}$ and $C_{\mathrm{C}}=0$. c A typical presentation in our series. The 2 volumes are partly overlapped, $1>D_{\mathrm{C}}$ and $C_{\mathrm{C}}>0 . D_{\mathrm{C}}$, Sørensen-Dice coefficient; $C_{\mathrm{C}}$, coverage coefficient.

by a neurosurgeon. The images were then imported to MATLAB ${ }^{\circledR}$ (2015b, MathWorks ${ }^{\mathrm{TM}}$, Inc., Natick, MA, USA) for rendering a 3D image, and the air volumes were segmented using the $i m 2 b w$ and graythreshold functions (intensity based). Positions of the segmented air volumes in MATLAB ${ }^{\circledR}$ were compared to the previously identified air by the neurosurgeon in order to exclude from the image the segmented structures that were not air. Region properties of the air volumes were then extracted and thereby number of pixels in the region multiplied by the pixel size in the 3 dimensions was used as air volume $(\mathrm{mL})$. An example of 1 air volume is visualized in Figure 1.

\section{Statistics}

The displacements in $x, y$, and $z$ for each side were checked for normal distribution (normplot, MATLAB, Mathworks, USA) and tested for statistical significance by paired, double-sided $t$ tests ( $t$ test, MATLAB Mathworks, USA). Values of $p<0.05$ were considered statistically significant. Unpaired, double-sided $t$ tests $(t$ test, Excel, Microsoft, USA) were performed to see whether the displacements on the left side were related to bilateral $(n=14)$ versus unilateral DBS, gender $(n=17$ male), diagnosis ET versus PD or age $<60$ years $(n=8)$.

Volumes, $V$, within the pulse width-dependent activation threshold $\mathrm{EF}_{\mathrm{t}}$ were exported as binary matrices with voxel sizes of $0.1 \times 0.1 \times 0.1 \mathrm{~mm}$ for calculation of the simulated volume, coverage coefficient, $C_{C}$ and the Sørensen-Dice coefficient, $D_{C}[35,36]$ between the acute and chronic simulations for each patient. The simulated volumes did not have a normal distribution and the paired Wilcoxon's signed rank test was used instead (signrank, MATLAB, Mathworks, USA) to compare simulated acute and chronic volumes when considering PES and not. In this study, $C_{\mathrm{C}}$ describes the common acute and chronic volume divided by the chronic volume. $D_{\mathrm{C}}$ describes the common acute and chronic volume divided by the acute and chronic volume (Fig. 2). $C_{\mathrm{C}}$ assumes 1 if the chronic volume is completely covered by the acute volume. $D_{\mathrm{C}}$ value of 1 means that the acute and chronic simulations have equal volume and position. Both $C_{C}$ and $D_{C}$ is 0 if there is no overlap between the 2 volumes (Fig. 2). Paired, double-sided $t$ tests
Table 2. Effects on left electrode displacement

\begin{tabular}{|c|c|c|}
\hline Parameter & $p$ value from $t$ test & $\begin{array}{l}\text { Difference, } \\
\mathrm{mm}\end{array}$ \\
\hline \multicolumn{3}{|c|}{ Double ( $n=14)$ vs. single-sided $(n=9)$ implantation } \\
\hline$x$ & ns & 0.05 \\
\hline$y$ & ns & 0.02 \\
\hline$z$ & ns & -0.04 \\
\hline \multicolumn{3}{|c|}{ Age $<60$ years $(n=8)$ vs. $\geq 60$ years $(n=15)$} \\
\hline$x$ & ns & 0.11 \\
\hline$y$ & ns & 0.37 \\
\hline$z$ & ns & -0.23 \\
\hline \multicolumn{3}{|c|}{ Gender man $(n=17)$ vs. woman $(n=6)$} \\
\hline$x$ & ns & 0.32 \\
\hline$y$ & ns & 0.19 \\
\hline$z$ & ns & -0.13 \\
\hline \multicolumn{3}{|c|}{$\mathrm{ET}(n=11)$ vs. $\mathrm{PD}(n=10)$} \\
\hline$x$ & ns & -0.41 \\
\hline$y$ & ns & -0.21 \\
\hline$z$ & ns & -0.16 \\
\hline \multicolumn{3}{|c|}{ Standard anchoring $(n=14)$ vs. Stimloc ${ }^{\mathrm{mm}}+\operatorname{Guardian}^{\mathrm{mm}}(n=9)$} \\
\hline$x$ & ns & -0.01 \\
\hline$y$ & ns & 0.16 \\
\hline$z$ & $<0.05$ & 0.63 \\
\hline
\end{tabular}

ET, essential tremor; PD, Parkinson's disease.

were done to compare the $C_{\mathrm{C}}$ value between acute and chronic assuming displacement versus no displacement, both for the simulations with PES and for the simulations without. The same $t$ test was performed when evaluating $D_{\mathrm{C}}$. Air volume was split in volumes for the left and the right brain hemisphere. Correlation between air volume and displacement was tested for $x, y$, and $z$ in each side (corrcoef, Matlab, Mathworks, USA).

\section{Results}

\section{Displacements}

The lead displacement tendencies were statistically significant on group level (Fig. 3). On average after approximately 1 month, the leads moved anterior $(y)$ and inferior $(z)$. The left side movements were (mean \pm SD) $y$ $(0.64 \pm 0.54, p<0.001)$ and $z(0.62 \pm 0.71, p<0.001)$ and corresponding values on the right side were $y(0.71 \pm 0.54$, $p<0.001)$ and $z(0.49 \pm 0.81, p<0.05)$. On the left side there was also a tendency for lateral displacement $x,(0.44$ $\pm 0.72, p<0.01$ ), while it was not significant on the right side $x(-0.11 \pm 0.61, \mathrm{~ns})$. The statistically significant contributing factor to the displacement was the anchoring technique and it reduced displacement in the $z$ direction (Table 2). 
Fig. 3. The boxplot (Matlab, Mathworks, USA) shows median values (red line), 25 and 75 percentiles (box), total range without outliers (dashed whiskers) and outliers (crosses). Directions of electrode movement in $x$ (medial-lateral, depending on the side), $y$ (anterior-posterior), $z$ (inferiorsuperior) for left side (a) and right side (b). The lead displacement was statistically significant in the direction anterior and inferior on both sides. The left side also showed a lateral displacement.

Fig. 4. The images display EF change due to lead movement and electric conductivity changes in the PES. Red is the acute volume, and blue is the chronic volume. The STN, axial view (a), coronal view (b). The $\mathrm{GPi}$, axial view (c), coronal view (d). EF, electric field; PES, perielectrode space; STN, subthalamic nucleus; GPi, globus pallidus internus.
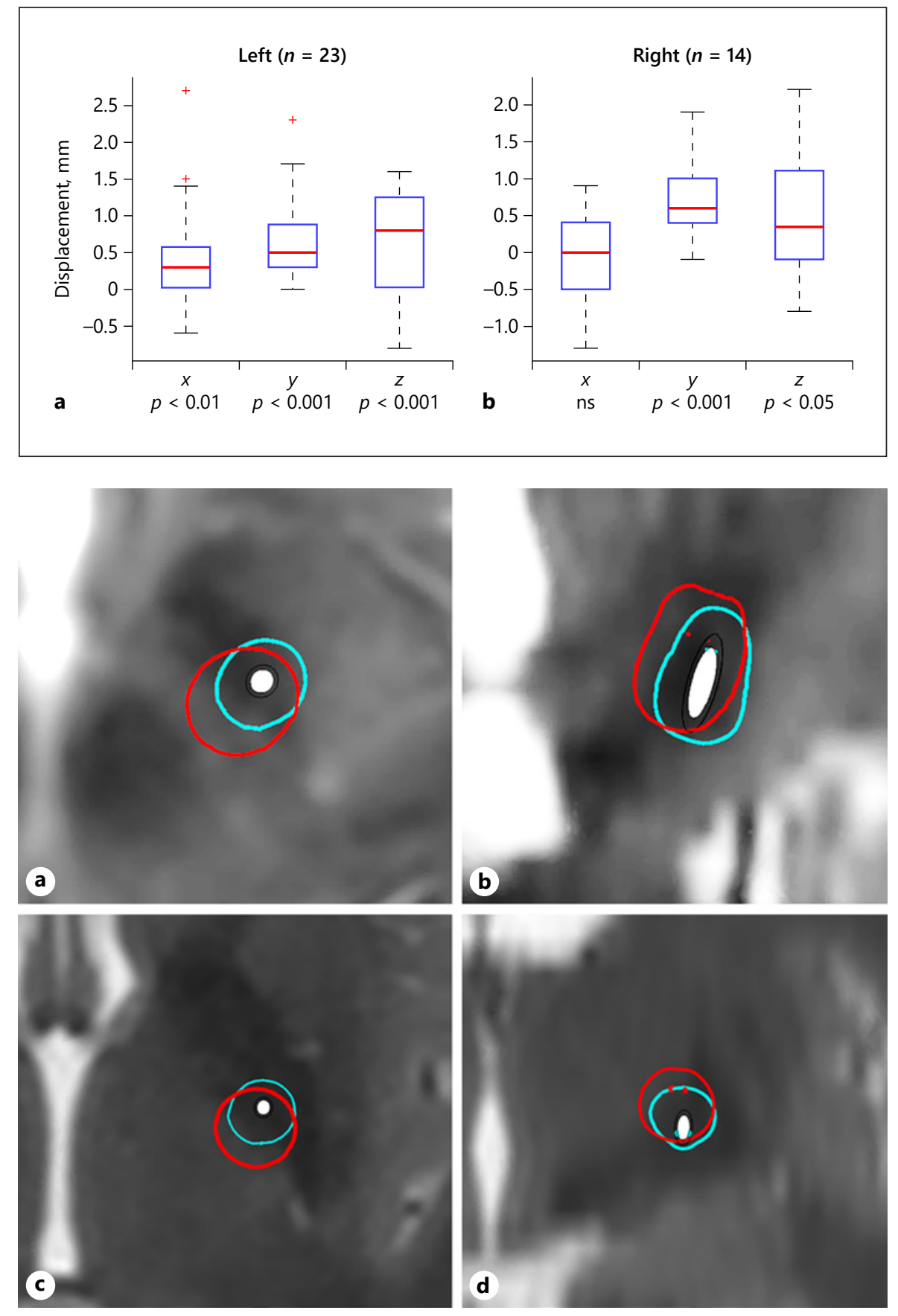

\section{Air Volume}

The median and range of the air volumes on the left and right side were $0.19(0-8.76)$ and $0.18(0-2.01) \mathrm{cm}^{3}$, respectively. The median and range of air volume for all 37 leads was $0.19(0-8.76) \mathrm{cm}^{3}$. There was no statistically significant correlation between calculated air volume and lead movement in any direction $(x, y$, and $z)$ for either hemisphere.

Postoperative Lead Movement after Deep Brain Stimulation Surgery

\section{Electric Field Simulations}

An example of change in EF due to lead movement and electric conductivity changes in the PES can be seen in Figure 4. The image shows the cumulative effect of both the typical electrode displacement (anterior and inferior) and the reduction of electric spread as the edema recedes with time and is replaced by gliosis. Thus, even though the EF shrinks, the displacement causes previ- 
Table 3. Acute and chronic stimulation volumes

\begin{tabular}{|c|c|c|}
\hline Parameter & With PES & Without PES \\
\hline Acute postoperative volume, $\mathrm{mm}^{3}$, median (25-75 percentiles) & $162(76-190)$ & $130(57-173)$ \\
\hline Chronic volume assuming displacement, $\mathrm{mm}^{3}$, median (25-75 percentiles) & $87(46-113)$ & $143(56-177)$ \\
\hline$p$ value, volume difference acute - chronic & $<0.001$ & ns \\
\hline$C_{\mathrm{C}}$ between acute and chronic assuming displacement, mean $\pm \mathrm{SD}$ & $0.72 \pm 0.18$ & $0.61 \pm 0.17$ \\
\hline$C_{\mathrm{C}}$ between acute and chronic, assuming no displacement & 1 & 1 \\
\hline$D_{\mathrm{C}}$ between acute and chronic, assuming no displacement, mean $\pm \mathrm{SD}$ & $0.74 \pm 0.06$ & 1 \\
\hline$p$ value, $D_{\mathrm{C}}$ difference, displacement - no displacement & $<0.001$ & $<0.001$ \\
\hline
\end{tabular}

PES, perielectrode space; $D_{\mathrm{C}}$, Sørensen-Dice coefficient; $C_{\mathrm{C}}$, coverage coefficient. $n=17$, bilateral: 6 , unilateral: 5 .

ously unaffected tissue to be affected. Results of the simulations are summarized in Table 3 together with statistical comparisons. The PES reduced the volume from 191 $\pm 174 \mathrm{~mm}^{3}$ in the acute stage to $111 \pm 101 \mathrm{~mm}^{3}$ in the chronic stage. This change was statistically significant at $p<0.001$. The $C_{C}$ when considering PES and when not considering PES between acute and chronic assuming displacement was $0.72 \pm 0.18$ and $0.61 \pm 0.17$ respectively. The impact of displacement on $C_{C}$ was statistically significant both with and without PES at $p<0.001$. The $D_{\mathrm{C}}$ when considering PES and without PES between acute and chronic assuming displacement was $0.54 \pm$ 0.14 and $0.61 \pm 0.17$. The $D_{\mathrm{C}}$ when considering PES between acute and chronic assuming no displacement was $0.74 \pm 0.06$. The impact of displacement on $D_{\mathrm{C}}$ was statistically significant both with and without PES at $p<$ 0.001 .

\section{Discussion}

The left lead tip displayed a tendency to move lateral, anterior, and inferior and the right a tendency to move anterior and inferior after 1 month. This small displacement was affected by the anchoring technique with Guardian and Stimlock covers on average giving a smaller displacement than the standard boxed cover. The PES changes with time and reduces the stimulation volume in the chronic stage compared to the acute. Despite this volume reduction, the electrode displacement gives a part of the stimulation volume in the chronic case that was not included in the acute phase, thus affecting new brain territory.

\section{Displacement}

Our experience is that if the DBS is initiated in the acute phase directly after surgery, the setting and chosen contact need to be changed within a short period. A lower voltage is needed for both clinical effect and to avoid side effects in the acute stage. This can partially be due to the microlesioning effect from the DBS lead itself which can be seen in up to $50 \%$ of the patients $[19,20]$. However, this observation can also be explained by postoperative lead displacement and changes of the electric conductivity. To our knowledge this study is the first to investigate postoperative lead displacement in addition to changes of the influence from PES and thus alteration of the electric conductivity after DBS. The lead displacement tendencies in our study after 1 month were small but statistically significant on group level (Fig. 3).

Co-fusion error, imaging resolution limitations, and brain pulsations could result in measured nonzero displacement values even if the electrode position is unchanged between the 2 points. The same method was utilized when analyzing the lead position at the 2 time points by using the identical imaging technique and protocol for co-fusion postoperatively. Any bias error in the coordinate estimation should thus be the same for the 2 time points and we should only have a variance error from the intrinsic imaging errors. When calculating absolute values, these random measurement errors may contribute to an overestimation of an average measured displacement. A previous study reported absolute confusion errors of about $0.5 \mathrm{~mm}$ which is in the range of the measured displacement in this study [37]. We, therefore, considered the displacement direction instead of investigating absolute values. By keeping the direction the intrinsic errors should only add to the variability of the displacement and 
any remaining significant difference in the $t$ test should be due to a real displacement on an average group level.

The lead tip displayed bilaterally a tendency to move anterior and inferior in the anatomy after approximately 1 month. A lateral displacement was seen for the left leads but not for the right. This discrepancy may be explained by that the lateral displacement is the smallest measured value and the right-side leads are fewer in number. Also, the $p$ value of the lateral displacement is, even though significant, weaker than the rest of the directions on the left side and therefore has the biggest risk of being a false positive value. In addition, it can be noted that the implantation of the lead was performed first on the left side for all bilateral surgeries and it cannot be discounted that this might affect the lateral displacement. Van den Munckhof et al. [9] and Sillay et al. [38] described an anterior and rostral movement. Kim et al. [8] suggest that the electrode position is stabilized 1 month after surgery and that the lead tip moves anterior and inferior with a measured discrepancy in accordance with our data. Considering that the target area is a few $\mathrm{mm}$ in size [13] and the quadripolar DBS lead consists of four 1.5-mm contacts and three 0.5 - $\mathrm{mm}$ interspaces, even a small displacement can theoretically have a clinical impact regardless of the direction of displacement. Further supporting this statement is that the side effects depend on the structures surrounding the target affected by the EF [2].

\section{Brain Shift}

Several studies discuss the role of brain shift on the electrode location. The direction of brain shift is hypothesized to move the brain laterally and caudally [38]. Van den Munckhof et al. [9] and Bentley et al. [10] describes that the shift is less in the deeper parts of the brain compared to the more superficial. When the brain shift resides, the brain tissue around the electrode contact can theoretically move with the electrode. Subsequently, the lead tip remains in the same anatomical position, but a new stereotactical position can be registered. Our data suggest that the displacement in itself represents an actual change in affected structures since the direction of displacement is inferior in addition to anterior and not superior as would be expected if the electrode followed the brain shift.

Previous studies have attributed displacement to technical errors, dystonia, twiddler syndrome, cerebrospinal fluid loss, pneumocephalus, and failure of lead fixation, $[8,9,11]$. We perform DBS surgery in 1 stage and thus the technical errors leading to dorsal migration of the lead associated with 2 stage implantation was not seen as de-

Postoperative Lead Movement after Deep

Brain Stimulation Surgery scribed by Morishita et al. [11]. The single patient with dystonia displayed a higher displacement than average. Twiddler syndrome was not seen during the period of this study.

\section{Correlation between Air and Lead Displacement}

Correlation between intracranial air (ICA) and lead displacement has previously been reported by van den Munckhof et al. [9] with a mean air volume to $17 \mathrm{~cm}^{3}$ with a max of $88 \mathrm{~cm}^{3}$. They showed that the ICA was correlated to an upward movement of the lead. Kim et al. [8] associated pneumocefalus to a difference in the $y$ coordinate of the lead tip coordinate with an estimated mean ICA of $11.9 \mathrm{~cm}^{3}$ with a max of $76 \mathrm{~cm}^{3}$. Bentley et al. [10] found that ICA was correlated to movement of the proximal part of the lead but not to the distal contact. Sillay et al. [38] had relatively lower ICA in comparison to the above mentioned studies and did not establish a correlation between pneumocefalus and displacement. The lesser air volume in our study may explain why no correlation was seen between ICA and lead movement in any direction $(x, y$, and $z)$ for either hemisphere.

\section{Anchoring Technique}

The only statistically significant variable found to be associated with displacement was the anchoring technique. Usage of Stimloc ${ }^{\mathrm{TM}}$ or Guardian ${ }^{\mathrm{TM}}$ reduced displacement in the inferior direction compared to using the standard anchoring cover that is boxed with the lead. This finding is supported by Contarino et al. [39] who correlated the anchoring technique to postoperative displacement after minimizing CSF leakage. No association was found between displacement and any of the other investigated factors: bilateral versus unilateral DBS, gender, and age $<60$ years or ET versus PD (Table 2). The results for the left side are presented in the case of bilateral placement since it would otherwise count as 2 independent observations for 1 patient when regarding the different factors.

\section{Perielectrode Space}

Evidence for increased conductivity from edema surrounding the electrode in the acute phase of DBS and reduced conductivity from gliosis in the chronic phase comes from impedance measurements over the first 20 weeks after implantation [31]. The edema surrounding the electrode is evident only in the acute phase after DBS implantation and has a higher conductivity compared to the gliosis-influenced chronic phase when using the same programming setting. The higher electric conductivity of 
the initial edema makes the EF spread further compared to the later gliosis formation, reducing the coverage of the initially affected volume. The simulated volume becomes smaller as the edema is replaced with gliosis which has lower electric conductivity. The conductivity properties will also be different if the lead has moved since the brain is heterogenic. This explains the small difference in volume also seen when not considering PES in the acute versus chronic phase since the lead movement was also considered when calculating the chronic volume. The edema, the gliosis, and the movement all can affect the size of the $\mathrm{EF}$ volume. However, the volume difference is only statistically significant in our study when including the effect of edema and gliosis in the PES (Table 3 ).

We show that even a small lead movement after DBS can be a factor to consider before starting the stimulation. The EF in the chronic stage is significantly lesser than the volume in the acute stage when considering PES. Therefore, the displaced chronic field may or may not be engulfed by the larger acute EF because of the size difference. Since the $C_{\mathrm{C}}$ is $<1$, the smaller chronic EF are partially situated outside the larger acute volumes (Fig. 2). The measured displacement indicates that new brain territory might be affected even when considering PES and this may be clinically important (Fig. 4). A $C_{\mathrm{C}}$ value of $0.72 \pm 0.18$ when considering PES corresponds to that $28 \%$ of the chronic volume is not overlapped by the acute volume. $D_{\mathrm{C}}$ considers both size and position. $D_{\mathrm{C}}$ without PES assuming displacement $(0.61 \pm 0.17)$ is smaller than $D_{\mathrm{C}}$ with PES assuming no displacement $(074 \pm 0.06)$. Thus, our data suggests that the displacement has a greater impact on $D_{C}$ than the PES (Table 3). It should be noted that the decrease in $D_{\mathrm{C}}$ due to displacement may be overestimated because of the previously mentioned limited image resolution. With this mentioned, our data suggests that the displacement has a greater impact on $D_{\mathrm{C}}$ than the PES (Table 3).

\section{Conclusion}

A programming setting that provides adequate symptom relief and avoids side effects is the goal after a properly placed electrode. Optimal programming is dependent on the final electrode location and brain structures stimulated. Using the Guardian ${ }^{\mathrm{TM}}$ and Stimlock ${ }^{\mathrm{TM}}$ as anchoring device seems better in reducing displacement in our series. Settings can change due to displacement and changes of PES. In some departments early postoperative programming to determine the amplitude threshold for clinical benefits and to determine side effects for each of the electrode contacts is performed direct postoperatively during the hospitalization period. The results in this study, however, suggest possible benefits with delayed programming at 4 weeks, in a more chronic state. New brain territory was affected due to the small displacement despite considering the reduced stimulated volume after 1 month. At this time the perielectrode edematic space has receded and the electrode is presumably fixated to its final position thus enhancing the possibilities for an optimal review of the contacts. This suggests that the patient comfort can be improved by providing a more correct and representative programming setting after 1 month compared to the acute phase. It also implies a cost beneficial aspect. Reducing excessive programming decreases the number of clinical visits to the hospital. The clinical implication by delaying the programming suggested by this paper can improve the treatment with DBS.

\section{Acknowledgement}

We would like to thank Neda Haj-Hosseini, PhD, Department of Biomedical Engineering, Linköping University for the air volume calculations.

\section{Statement of Ethics}

The study was approved by the local Ethics Committee (2012/434-31) and all patients gave their informed written consent to participate in the study.

\section{Conflict of Interest Statement}

The authors have no conflicts of interest to declare. K.W. has shares in FluoLink AB, Sweden K.W. has shares in FluoLink AB, Sweden.

\section{Funding Sources}

The study was supported by the Swedish Research Council (2016-03,564), Swedish Foundation for Strategic Research (BD150032) and by ALF Grants Region Östergötland (LIO-698041).

\section{Author Contributions}

N.G. performed the image analysis and the statistics. J.D.J. performed the FEM simulations and the statistics. N.G., J.D.J., K.W., and P.Z. all contributed to the study design and writing. 


\section{References}

1 Lozano AM. Vim thalamic stimulation for tremor. Arch Med Res. 2000 May-Jun;31(3): 266-9.

2 Benabid AL, Chabardes S, Mitrofanis J, Pollak P. Deep brain stimulation of the subthalamic nucleus for the treatment of Parkinson's disease. Lancet Neurol. 2009 Jan;8(1):67-81.

3 Hariz M, Blomstedt P, Zrinzo L. Future of brain stimulation: new targets, new indications, new technology. Mov Disord. 2013 Nov;28(13): 1784-92.

4 Greenberg BD, Gabriels LA, Malone DA Jr, Rezai AR, Friehs GM, Okun MS, et al. Deep brain stimulation of the ventral internal capsule/ventral striatum for obsessive-compulsive disorder: worldwide experience. $\mathrm{Mol}$ Psychiatry. 2010 Jan;15(1):64-79.

5 Khan MF, Mewes K, Gross RE, Skrinjar O. Assessment of brain shift related to deep brain stimulation surgery. Stereotact Funct Neurosurg. 2008;86(1):44-53.

6 Paek SH, Yun JY, Song SW, Kim IK, Hwang $\mathrm{JH}$, Kim JW, et al. The clinical impact of precise electrode positioning in STN DBS on three-year outcomes. J Neurol Sci. 2013 Apr 15;327(1-2):25-31.

7 Matias CM, Frizon LA, Asfahan F, Uribe JD, Machado AG. Brain shift and pneumocephalus assessment during frame-based deep brain stimulation implantation with intraoperative magnetic resonance imaging. Oper Neurosurg. 2018 Jun 1;14(6):668-74.

8 Kim YH, Kim HJ, Kim C, Kim DG, Jeon BS, Paek SH. Comparison of electrode location between immediate postoperative day and 6 months after bilateral subthalamic nucleus deep brain stimulation. Acta Neurochir. 2010 Dec;152(12):2037-45.

9 van den Munckhof P, Contarino MF, Bour LJ, Speelman JD, de Bie RM, Schuurman PR. Postoperative curving and upward displacement of deep brain stimulation electrodes caused by brain shift. Neurosurgery. 2010 Jul; 67(1):49-4.

10 Bentley JN, Guan Z, Cummings KS, Chou KL, Patil PG. Influence of intracranial air on electrode position and clinical outcomes following deep brain stimulation for Parkinson's disease. Stereotact Funct Neurosurg. 2017; 95(1):6-12.

11 Morishita T, Hilliard JD, Okun MS, Neal D, Nestor KA, Peace D, et al. Postoperative lead migration in deep brain stimulation surgery: incidence, risk factors, and clinical impact. PLoS One. 2017;12(9):e0183711.

12 Aziz TZ, Nandi D, Parkin S, Liu X, Giladi N, Bain $\mathrm{P}$, et al. Targeting the subthalamic nucleus. Stereotact Funct Neurosurg. 2001;77(14):87-90.

13 Richter EO, Hoque T, Halliday W, Lozano AM, Saint-Cyr JA. Determining the position and size of the subthalamic nucleus based on magnetic resonance imaging results in patients with advanced Parkinson disease. Neurosurg. 2004 Mar;100(3):541-6.
14 Åström M, Zrinzo LU, Tisch S, Tripoliti E, Hariz MI, Wårdell K. Method for patient-specific finite element modeling and simulation of deep brain stimulation. Med Biol Eng Comput. 2009 Jan;47(1):21-8.

15 Alonso F, Latorre MA, Göransson N, Zsigmond $\mathrm{P}$, Wårdell K. Investigation into deep brain stimulation lead designs: a Patient-Specific Simulation Study. Brain Sci. 2016 Sep 7;6(3):39.

16 Åström M, Tripoliti E, Hariz MI, Zrinzo LU, Martinez-Torres I, Limousin P, et al. Patientspecific model-based investigation of speech intelligibility and movement during deep brain stimulation. Stereot Funct Neuros. 2010;88(4):224-33.

17 Hemm S, Pison D, Alonso F, Shah A, Coste J, Lemaire JJ, et al. Patient-specific electric field simulations and acceleration measurements for objective analysis of intraoperative stimulation tests in the thalamus. Front Hum Neurosci. 2016;10:577.

18 Akbarian-Tefaghi L, Akram H, Johansson J, Zrinzo L, Kefalopoulou Z, Limousin P, et al. Refining the deep brain stimulation target within the limbic globus pallidus internus for tourette syndrome. Stereotact Funct Neurosurg. 2017;95(4):251-8.

19 Maltete D, Derrey S, Chastan N, Debono B, Gerardin E, Freger P, et al. Microsubthalamotomy: an immediate predictor of longterm subthalamic stimulation efficacy in Parkinson disease. Mov Disord. 2008 May 15; 23(7):1047-50.

20 Tykocki T, Nauman P, Koziara H, Mandat T. Microlesion effect as a predictor of the effectiveness of subthalamic deep brain stimulation for Parkinson's disease. Stereotact Funct Neurosurg. 2013;91(1):12-7.

21 Yousif N, Bayford R, Wang S, Liu X. Quantifying the effects of the electrode-brain interface on the crossing electric currents in deep brain recording and stimulation. Neuroscience. 2008 Mar 27;152(3):683-91.

22 Alonso F, Hemm-Ode S, Wårdell K. Influence on deep brain stimulation from lead design, operating mode and tissue impedance changes: a simulation study. Brain Disord Ther. $2015 ; 4(3)$

23 Johansson JD, Alonso F, Wårdell K. Modelling details for electric field simulations of deep brain stimulation. Prague; 2018.

24 Åström M, Johansson JD, Hariz MI, Eriksson $\mathrm{O}$, Wårdell K. The effect of cystic cavities on deep brain stimulation in the basal ganglia: a simulation-based study. J Neural Eng. 2006 Jun;3(2):132-8.

25 Wårdell K, Hemm-Ode S, Rejmstad P, Zsigmond P. High-resolution laser doppler measurements of microcirculation in the deep brain structures: a method for potential vessel tracking. Stereotact Funct Neurosurg. 2016; 94(1):1-9.

26 Blomstedt P. Implanting a DBS system. In: Blomstedt P, Hariz M, editors. Encyclopedia Stereotactica. A practical guide to stereotactic functional neurosurgery. 2017. www.stereotactic.org.

27 Johansson JD, Alonso F, Wårdell K. Patientspecific simulations of deep brain stimulation electric field with aid of In-house software ELMA. Berlin. 2019.

28 Wårdell K, Zrinzo L, Hariz M, Andersson M. Patient-specific brain modelling for deep brain stimulation simulations. I IEEE Embs C Neur E. 2013:148-51.

29 Gabriel S, Lau RW, Gabriel C. The dielectric properties of biological tissues: III. Parametric models for the dielectric spectrum of tissues. Phys Med Biol. 1996 Nov;41(11):2271-93.

30 Andreucetti D, Fossi R, Petrucci C. An Internet resource for the calculation of the dielectric properties of body tissues in the frequency range $10 \mathrm{~Hz}-100 \mathrm{GHz}$. Florence, Italy: IFACCNR; 1997.

31 Lungu C, Malone P, Wu T, Ghosh P, McElroy B, Zaghloul K, et al. Temporal macrodynamics and microdynamics of the postoperative impedance at the tissue-electrode interface in deep brain stimulation patients. J Neurol Neurosurg Psychiatry. 2014 Jul;85(7):816-9.

32 Rizzone M, Lanotte M, Bergamasco B, Tavella A, Torre E, Faccani G, et al. Deep brain stimulation of the subthalamic nucleus in Parkinson's disease: effects of variation in stimulation parameters. J Neurol Neurosurg Psychiatry. 2001 Aug;71(2):215-9.

33 Kuncel AM, Cooper SE, Grill WM. A method to estimate the spatial extent of activation in thalamic deep brain stimulation. Clin Neurophysiol. 2008 Sep;119(9):2148-58.

34 Åström M, Diczfalusy E, Martens H, Wårdell K. Relationship between neural activation and electric field distribution during deep brain stimulation. IEEE Trans Biomed Eng. 2015;62(2):664-72.

35 Dice LR. Measures of the amount of ecologic association between species. Ecology. 1945; 26(3):297-302.

36 Alonso F, Vogel D, Johansson J, Wårdell K, Hemm S. Electric field comparison between microelectrode recording and deep brain stimulation systems-a simulation study. Brain Sci. 2018 Feb 6;8(2).

37 Geevarghese R, O'Gorman Tuura R, Lumsden DE, Samuel M, Ashkan K. Registration accuracy of CT/MRI fusion for localisation of deep brain stimulation electrode position: an imaging study and systematic review. Stereotact Funct Neurosurg. 2016;94(3):159-63.

38 Sillay KA, Kumbier LM, Ross C, Brady M, Alexander A, Gupta A, et al. Perioperative brain shift and deep brain stimulating electrode deformation analysis: implications for rigid and non-rigid devices. Ann Biomed Eng. 2013 Feb;41(2):293-304.

39 Contarino MF, Bot M, Speelman JD, de Bie RM, Tijssen MA, Denys D, et al. Postoperative displacement of deep brain stimulation electrodes related to lead-anchoring technique. Neurosurgery. 2013 Oct;73(4):681-188. 